\title{
Enfermedad de Chagas
}

\section{EPIDEMIOLOGía}

\section{Seguimiento serológico de hijos de madres seropositivas a Trypanosoma cruzi en la Jurisdicción Sanitaria de Tuxpan, Veracruz, México}

Adela Ruiz¹, Paz María Salazar ${ }^{1}$, Gloria Rojas ${ }^{1}$, Yolanda Guevara1', Elia Torres¹, Manuel Gutiérrez¹, Leticia Ruiz¹, Isabel Prichi ${ }^{2}$, Manuel Flores ${ }^{2}$

1 Facultad de Medicina, Universidad Nacional

Autónoma de México, México, D.F., México

2 Servicios de Salud del Estado de Veracruz, México

Introducción. Un estudio previo sobre la enfermedad de Chagas en Veracruz, encontró una seroprevalencia de 2,8 \%, en individuos de la Jurisdicción Sanitaria 2, Tuxpan. Derivado de estos resultados, se realizó otro estudio con el fin de detectar la transmisión vertical y determinar, mediante seguimiento serológico, la presencia o ausencia de anticuerpos anti-Trypanosoma cruzi, en hijos de madres seropositivas del mismo lugar, confirmadas por ELISA e IFI,.

Si T. cruzi puede transmitirse por vía materna, entonces, es probable que el producto se infecte por esta vía. Considerando esta posibilidad y asumiendo que la salud de los recién nacidos puede mejorar si la enfermedad es diagnosticada y tratada en el primer año de vida, es importante realizar estudios de seguimiento serológico en los hijos de mujeres embarazadas positivas para $T$. cruzi.

Materiales y métodos. Entre 2001 y 2010 se estudiaron 4.851 mujeres embarazadas de consulta prenatal; de éstas, se seleccionaron 20 (0,41\%) seropositivas, confirmadas y con seguimiento por ELISA e IFI; se estudiaron serológicamente veinte hijos al nacimiento y posteriormente se les hizo seguimiento serológico hasta los 2 años de edad.

Resultados. La complejidad del trabajo en comunidad permitió realizar desde 1 hasta 9 determinaciones serológicas; los resultados serológicos fueron negativos para T. cruzi en 16 casos, empleando ambas pruebas. En cuatro niños sólo fue posible realizar en una ocasión las pruebas. Todos los casos nacidos positivos a $T$. cruzi, fueron negativos al cabo de los 2 años.

Conclusiones. No se encontraron anticuerpos en los hijos de madres seropositivas después de 6 meses del nacimiento. La tasa de prevalencia en mujeres gestantes con enfermedad con Chagas en la zona de estudio es de $0,41 \%$ y de $0 \%$ de incidencia de transmisión vertical; contrario a lo que sucede en Bolivia, Paraguay, Brasil o Argentina. Se desconoce el grado de endemia en la región estudiada y las cepas del parásito presentes.

\section{Miocardiopatía dilatada y enfermedad de Chagas: reporte de un caso en Ecuador}

Ángel Guevara, Fernando Cisneros, Gabriel Runruil, Manuel Calvopiña, Yosselin Vicuña, Carmen Perugachi Centro de Biomedicina, Laboratorio de Parasitología Molecular, Escuela de Medicina, Universidad Central del Ecuador; Hospital Eugenio Espejo, Quito, Ecuador

Introducción. La enfermedad de Chagas, o tripanosomiasis americana, causada por Trypanosoma cruzipresenta tres fases: aguda, en la que los niveles de parasitemia pueden estar elevados y se podría detectar el parásito por microscopía; en las fases intermedia, o indeterminada, y crónica casi no hay parásitos circulantes y pueden aparecer patologías asociadas, como megavísceras. La mayoría de las infecciones agudas no se diagnostican y la enfermedad clínica suele manifestarse años después durante la fase crónica.

En el presente trabajo se comprobó infección por Trypanosoma cruzi mediante serología y reacción en cadena de la polimerasa (PCR) en una paciente ecuatoriana con miocardiopatía dilatada.

Materiales y métodos. Se presenta el caso de un paciente, de sexo femenino y 30 años de edad, que consultó por disnea de pequeños esfuerzos; se practicó examen clínico completo, análisis de laboratorio, imégenes y cardiología. Se ordenaron pruebas inmunológicas en sangre para anticuerpos anti-Trypanosoma cruzi y PCR para amplificar ADN específico de T. cruzi.

Resultados. El examen físico y clínico revela ruidos cardíacos arrítmicos, soplo holosistólico con regurgitación de grado III, ligera hepatomegalia, hipotensión postural, arritmias ventriculares, taquicardias y fibrilaciones ventriculares. En los análisis rutinarios de laboratorio no se encontraron alteraciones; los anticuerpos IgG anti-T.cruzifueron positivos para todas las pruebas inmunológicas y la 
PCR para T. cruzi fue también positiva. El análisis de imágenes demostraron una cardiomegalia de grado IV. En el estudio electrocardiográfico se encontró ritmo de escape ventricular y bloqueo de rama derecha. En la ecografía se observó dilatación moderada de los ventrículos e importante de aurícula derecha.

Conclusiones. En el presente caso, la valoración clínica determinó una cardiomiopatía dilatada con arritmia ventricular y diagnóstico funcional de insuficiencia cardiaca que facilitó el tratamiento adecuado del paciente. Las pruebas inmunológicas y moleculares demostraron la presencia de infección por T. cruzi en un caso de cardiomiopatía dilatada que debe alertar a los servicios de cardiología para considerar como causa etiológica del problema cardiaco idiopático la infección por $T$. cruzi.

Es necesario continuar con los programas de detección, prevención y educación a la comunidad de áreas endémicas y no endémicas para la enfermedad de Chagas.

Con el auspicio de Consejo Superior de Investigaciones Científicas de España y proyecto RedTrV (CYTED 209RT0364).

$$
\bullet \bullet
$$

\section{Supervivencia de Trypanosoma cruzi en bebidas experimentalmente contaminadas}

Diana Carolina Suárez, Ángela Patricia Rey, Magda Lorena Orduz, Renzo Leonardo Prada, Zorayda

Tarazona de Ramírez

Bucaramanga, Colombia

Introducción. Trypanosoma cruzi es el agente causante de la enfermedad de Chagas, el cual puede ser transmitido por diferentes vías. A partir del 2005 la transmisión oral incrementó en los países donde la enfermedad es considerada endémica por transmisión vectorial.Colombia no se aparta de esta tendencia, situación que motivó la alerta epidemiológica, urgiendo la necesidad de encontrar explicación a la infección oral de la parasitosis.

Objetivo. Este estudio busca establecer registros del tiempo de supervivencia de una cepa de Trypanosoma cruzi en los jugos implicados en el brote del municipio de Lebrija en el 2008.

Materiales y métodos. Se evaluó la supervivencia, definida por los criterios de vitalidad (movimiento progresivo) y viabilidad (aislamiento en medio de cultivo NNN/LIT) de la cepa de T. cruzi aislada de un paciente del brote del municipio de Aguachica, en jugos de mandarina, guayaba, guanábana, agua y agua con azúcar.
Resultados. La cepa T. cruzi DS presentó vitalidad en jugo de mandarina a temperatura ambiente, 72 horas, en refrigeración de 36 horas; en jugo de guanábana a temperatura ambiente, 48 horas, en refrigeración de 384 horas, y en jugo de guayaba en las dos temperaturas 24 horas; en el agua con azúcar se mantuvo vital hasta 240 horas a temperatura ambiente y 120 horas a temperatura de refrigeración. Esta cepa fue viable 2 y 24 horas después de la contaminación en cada una de las bebidas a las temperaturas estudiadas. En las condiciones de estudio la cepa no sobrevivió en agua del acueducto de Bucaramanga.

Conclusiones. Trypanosoma cruzi, cepa DS, sobrevivió en todas las bebidas por más de 24 horas después de la contaminación; se observó un tiempo de supervivencia de 384 horas en el jugo de guanábana; este hecho fortalece la hipótesis de la transmisión por vía oral. La cepa no sobrevivió en agua del acueducto de Bucaramanga, con lo que disminuye la posibilidad de considerar brotes de origen hídrico.

\section{Transmisión oral en modelo en ratón por jugos contaminados con Trypanosoma cruzi}

Diana Carolina Suárez, Ángela Patricia Rey, Magda Lorena Orduz, Zorayda Tarazona de Ramírez Bucaramanga, Colombia

Introducción. La enfermedad de Chagas es una infección parasitaria causada por el hemoflagelado Trypanosoma cruzi. En Colombia, del 2008 al 2010 se notificaron varios microbrotes de la enfermedad; el compromiso de grupos de personas enfermas simultáneamente, la ausencia conjunta de factores de riesgo de infección vectorial y la posibilidad de transmisión oral, nos conllevó a plantear si T. cruzi se podía transmitir por esta vía, la cual se ha visto asociada al consumo de alimentos contaminados, partiendo de los hechos ocurridos en el municipio de Lebrija (Santander), en los que se presume que los jugos fueron la fuente de infección de dicho brote.

Objetivo. Comprobar la probabilidad de infección oral, usando jugos contaminados experimentalmente con $T$. cruzi en un modelo de ratón.

Materiales y métodos. Se contaminaron 30 ratones con los jugos (guayaba, guanábana y mandarina) más la cepa DS de $T$. cruzi; se mantuvieron en observación durante 40 días; finalizado el periodo de observación, se les practicó la eutanasia; posteriormente, se practicaron los estudios anatomopatológicos e histológicos, y se 
hizo cultivo de corazón e hígado en medio bifásico NNN/LIT de cada ratón para posible aislamiento del parásito.

Resultados. El curso de la enfermedad fue similar al cuadro clínico de los pacientes involucrados en los brotes; macroscópicamente, se observó inflamación de los órganos analizados, acompañados de hallazgo de nidos de amastigotes en corazón y estómago; en los demás tejidos se observó abundante reacción inflamatoria.

Conclusiones. Se comprobó la transmisión del parásito $T$. cruzi por vía oral en el modelo de ratón, sustentando la hipótesis que explicó la causa de los microbrotes que se registraron desde 2008 hasta 2010 en la región nororiental de Colombia.

\section{Prevalencia de anticuerpos para Trypanosoma cruzi en perros de dos municipios endémicos de Boyacá}

Diego Alexander Manrique-Abril, Fred G. ManriqueAbril, Myriam Lorca, Juan Manuel Ospina

Grupo de investigación en Salud Pública, Universidad Pedagógica y Tecnológica de Colombia, Tunja, Boyacá, Colombia; Laboratorio Clínico CAMPVS, Santiago de Chile, Chile

Introducción. Laconvivencia con perros representa significativos riesgos, en la medida en que las condiciones ambientales, particularmente en zonas endémicas, favorecen múltiples infestaciones tanto por insectos vectores como por parásitos, lo que disminuye en el caso de la enfermedad de Chagas, la eficacia de las acciones de rociado con insecticidas de las casas como medida preventiva del control de los vectores.

El objetivo del estudio fue evaluar la prevalencia de anticuerpos anti Trypanosoma cruzi en una muestra de perros domésticos residentes en dos municipios endémicos.

Materiales y métodos. Se tomaron muestras séricas de 20 perros procedentes de hogares donde residían mujeres gestantes seropositivas para la enfermedad de Chagas y 40 perros de hogares de mujeres gestantes seronegativas de Miraflores y Moniquirá, Boyacá. El análisis se realizó mediante prueba diagnóstica rápida dipstick de InBios.

Resultados. Se encontró una prevalencia de $16,7 \%$ en Moniquirá y de $13,3 \%$ en Miraflores, con una prevalencia general de $15 \%$ en los dos municipios. Se halló un riesgo tres veces mayor de que ocurriera la infección en perros, en los hogares donde residían mujeres gestantes seropositivas; además, la infestación por pulgas y garrapatas en el animal, y el hábitat cercano a la vivienda, se relacionaron con mayores resultados seropositivos en el perro.

Conclusión. La raza, el sexo, la presencia de aves en la casa y al examen clínico general son considerados factores pronósticos en en la infección por Tyipanosoma cruzi en caninos. Como factores protectores se identificó la desparasitación y vacunación de los animales.

\section{Caracterización molecular de} Trypanosoma cruzi aislado en microbrotes de Chagas agudo presentados en el Departamento de

\section{Santander}

Dora Milena Villamil, Martha Lucía Díaz, Clara Isabel

González

Grupo de Inmunología y Epidemiología Molecular, Universidad Industrial de Santander, Bucaramanga, Colombia

Introducción. Trypanosoma cruzi es el agente causal de la enfermedad de Chagas, un importante problema de salud pública en todo el continente americano. En Colombia, $5 \%$ de la población está infectada y $20 \%$ está en riesgo de infección. Actualmente, $T$. cruzi se distribuye en 6 unidades discretas de tipificación, de Tcl a TcVI, y Tcl y II son los ancestrales. En Colombia, la principal forma de presentación clínica de la enfermedad de Chagas es la cardiomiopatía chagásica crónica. Sin embargo, en el departamento de Santander en los últimos tres años se han presentado seis brotes agudos de enfermedad de Chagas de probable transmisión oral con tres casos fatales.

Dados lo inusual de la presentación clínica, la seriedad y la mortalidad observada en los brotes, en este estudio caracterizamos genéticamente los aislamients obtenidos de pacientes con Chagas agudo de probable transmisión oral, que se presentaron en la zona urbana y rural de baja endemia en el departamento de Santander.

Materiales y métodos. Se caracterizaron molecularmente 13 aislamientos por amplificación de los genes miniexón, subunidad $18 \mathrm{~S}$ y $24 \mathrm{~S}$ del rRNA, citocromo oxidasa II, citocromo b y los loci HSP60 (proteína de choque térmico) y GPI (glucosa-6-fosfato isomerasa).

Resultados. La caracterización genética de los aislamientos por amplificación de los diferentes marcadores moleculares presentó resultados concordantes entre ellos y demostró que todos los aislamientos pertenecían al grupo Tc I. 
Conclusiones. Los casos agudos de enfermedad de Chagas reportados en diferentes municipios del departamento de Santander fueron causados por T. cruzi I, lo cual permite corroborar el predominio de este grupo en la región, asociado a los casos agudos y a los crónicos graves. Dada la variabilidad genética reportada para este grupo, actualmente se encuentra en proceso la clasificación por haplotipos.

\section{Seroprevalencia de la infección con Trypanosoma cruzi en perros de la zona urbana y rural del municipio de Talaigua Nuevo, departamento de Bolívar}

Edilson Garcés ${ }^{1}$, Luis Cortés², Ramiro Pereira ${ }^{3}$, Omar

Triana ${ }^{1}$, Omar Cantillo ${ }^{1}$

1 Grupo Biología y Control de Enfermedades Infecciosas, Universidad de Antioquia, Medellín, Colombia

2 Unidad de Entomología Médica, Laboratorio Departamental de Salud Pública, Departamento de Bolívar Cartagena, Colombia

3 Laboratorio Departamental de Salud Pública, Departamento de Bolívar, Cartagena, Colombia

Introducción. La vigilancia entomológica de triatominos llevada a cabo en el municipio de Talaigua Nuevo durante el 2010 y el primer semestre del 2011, mostró infecciones cercanas al $40 \%$ en Triatoma maculata peridoméstico. Sin embargo, la vigilancia serológica realizada a residentes y población infantil expuesta, no mostró transmisión en humanos. Por lo tanto, llama la atención la fuente de infección de $T$. maculata y su capacidad para producir focos de trasmisión.

Objetivo. Determinar la seroprevalecia de la infección con $T$. cruzi en perros de la zona rural y urbana de Talaigua Nuevo, Bolívar.

Materiales y métodos. El estudio se adelantó en la cabecera municipal de Talaigua Nuevo y en los corregimientos de Talaigua Viejo y El Vesubio. Se realizó un muestreo aleatorio estratificado, con un tamaño muestral de 164 perros. Sólo se incluyeron perros menores dos años o que durante este tiempo hubiesen vivido en la zona. Para la toma de $4 \mathrm{ml}$ de sangre total de la vena safena, se contó con la aprobación y ayuda del propietario. Todos los sueros obtenidos se procesaron por la técnica ELISA y confirmados por IFI, con un punto de corte igual a 1:40. Los sueros positivos en ambas técnicas se consideraron seropositivos.

Resultados. Se muestrearon 148 perros, el $90 \%$ del tamaño calculado. La prevalencia de anticuerpos anti- $T$. cruzi en sueros fue de 19,5\% (29/148). Para la cabecera de Talaigua Nuevo la prevalencia fue de $25 \%$ (19/76), mientras que para la zona rural de 13,9\% (10/72). La distribución de los perros infectados, en parte, se superpone con la distribución de T. maculata infectados por $T$. cruzi.

Conclusión. La alta seroprevalencia canina en la zona sugiere una gran dinámica de trasmisión entre perros y $T$. maculata, sin embargo, el papel de los perros como reservorio o barrera de infección a los residentes debe ser confirmado en futuras investigaciones.

\section{Anticuerpos anti-Trypanosoma cruzi en comunidades indígenas de los estados Bolívar y Delta Amacuro}

Elizabeth Askew, Julman Cermeño, Freddy Salazar Venezuela

Introducción. La enfermedad de Chagas, o tripanosomiasis americana, es una endemia que afecta a millones de personas, causando problemas sanitarios, económicosysociales. Tradicionalmente, el estado Bolívar y Delta Amacuro es considerado como no endémico de enfermedad de Chagas, a pesar de que existen las condiciones propicias para la transmisión.

El objetivo de este estudio fue determinar la prevalencia de anticuerpos anti- $T$. cruzi en tres comunidades indígenas (eñepa, hiwi y warao) de los estados Bolívar y Delta Amacuro, Venezuela.

Materiales y métodos. Se realizó un estudio prospectivo. Previo consentimiento informado para participar en el estudio de forma voluntaria, se aplicó una encuesta para la recolección de datos clínicos y epidemiológicos. Se tomaron muestras de sangre venosa para la obtención de suero y se determinó la presencia de anticuerpos específicos (IgG) contra T. cruzi mediante ELISA (BiosChile).

Resultados. Se evaluaron 159 individuos, de los cuales, 2,52 \% $(n=4)$ resultó seropositivo para la infección con $T$. cruzi. Al grupo de pacientes positivos, se le practicaron tres pruebas adicionales (Chagatest HAI, Chagatest ELISA y Chagatest Látex de Wiener Lab) para confirmar la presencia de anticuerpos, resultando los cuatro pacientes positivos; la población femenina fue de $0,01 \%$ y en la población masculina, de 0,04 \%y pertenecían en su totalidad a la etnia eñepa (municipio Cedeño, Estado Bolívar).

Conclusión. Este estudio demuestra la presencia de individuos infectados y la transmisión activa de 
la enfermedad de Chagas en el municipio Cedeño del Estado Bolívar.

\section{Detección y caracterización de las unidades discretas de tipificación de Trypanosoma cruzi que circula en comunidades del Chaco boliviano}

Esdenka Pérez, Marcelo Monje, Rudy Parrado, Lineth García, François Noireau, Simone Frédérique Brenière MIVEGEC (UM1 et 2-CNRS 5290- IRD 224) IRD Representación en Bolivia, La Paz; Instituto Nacional de Laboratorios de Salud (INLASA), Laboratorio de Entomología Médica, La Paz, Bolivia; IIBISMED, Facultad de Medicina, Universidad Mayor de San Simón, Cochabamba, Bolivia

Introducción. El Gran Chaco es una región hiperendémica para la enfermedad de Chagas, y Triatoma infestans el el principal vector de Trypanosoma cruzi. Lagran variedad intraespecífica de T. cruzi (6 unidades discretas de tipificación, Tcl a TcVI) hace necesaria su caracterización para conocer mejor las condiciones de transmisión.

El objetivo del estudio fue detectar e identificar las unidades discretas de tipificación de T. cruzi en $T$. infestans domésticos y peridomésticos en cuatro comunidades del Izozog, departamento de Santa Cruz, Bolivia.

Materiales y métodos. Se detectaron las unidades discretas de tipificación en $160 \mathrm{~T}$. infestans capturados antes y después del rociado. Después de la extracción de ADN a partir de las heces y del contenido estomacal se aplicó la prueba de PCR múltiplex miniexón, la cual distingue tres grupos de unidades discretas de tipificación: Tcl; Tcll, TcV y TcVI, y Tclll y TclV.

Resultados. Antes del rociado (84 insectos recolectados en 72 habitaciones), la tasa de infección fue de 40 \% y la mayoría de los insectos estuvieron infectados por unidades discretas de tipificación del grupo "Tcll, TcV y TcVI" (70 \%). La unidad discreta de tipificación Tcl se detectóen 21 $\%$ de los insectos, y del grupo de unidades discretas de tipificación "Tclll y TcIV" en (17\%). Después del rociado (76 determinaciones, procedentes de 44 habitaciones), la tasa de infección fue de $48 \%$. No se observaron diferencias significativas en la distribución de las unidades discretas de tipificación antes del rociado y después de él entre los insectos capturados en el peridomicilio o en el domicilio. De forma similar, no se observaron diferencias entre machos y hembras. En 13 insectos (8\%), se observó una mezcla de grupos de unidades discretas de tipificación.
Conclusiones. La presencia de los tres grupos de unidades discretas de tipificación en las comunidades del lzozog es una observación única, que da particularidades epidemiológicas propias a la región del Chaco Boreal en Bolivia. El mantenimiento de una alta tasa de infección y lan gran diversidad del parásito en los vectores después del rociado, se relaciona posiblemente con el fracaso del rociado y la rápida reinfestación que se observa en la zona.

\section{Transmisión enzoótica de Trypanosoma cruzi en Caracas, Distrito Capital, Venezuela}

H. J. Carrasco ${ }^{1}$, F. Alfonso², H. Montañez ${ }^{2}$, M.

Rodríguez¹, A. Pomenta ${ }^{1}$, D. Ugueto ${ }^{1}$, J.C. Londoño ${ }^{1}$, C. Martínez ${ }^{2}$

1 Laboratorio de Biología Molecular de Protozoarios, Instituto de Medicina Tropical, Universidad Central de Venezuela, Caracas, Venezuela

2 Dirección General de Salud Ambiental, Ministerio del Poder Popular para la Salud

Introducción. El presente trabajo permitió dilucidar qué huésped hace posible el mantenimiento del ciclo biológico de Trypanosoma cruzi, protozoo causante de la enfermedad de Chagas, en asociación con el triatomino Panstrongylus geniculatus, en un ciclo principalmente selvático, pero encontrado también en áreas urbanas de Caracas.

El objetivo del estudio fue determinar los componentes de la transmisión enzoótica de $T$. cruzi en Caracas, Venezuela.

Materiales y métodos. Se evaluaron 95 ratas (Rattus rattus) provenientes de la parroquia La Pastora y 89 triatominos de la especie $P$. geniculatus recolectados en las parroquias La Pastora, Altagracia y San José. Se realizó diagnóstico parasicológico en ratas y triatominos e infección artificial en modelo de ratón. Los parásitos obtenidos por infección artificial se cultivaron en medio agar-sangre y medio RPMI. La caracterización genotípica de los aislamientos de T. cruzi se realizó con la técnica de ADN polimorfo amplificado aleatoriamente (RAPD).

Resultados. El $41 \%$ de las ratas resultaron positivas para $T$. cruzi y el genotipo circulante del parásito fue tipificado como Tcl. De igual manera, el $60,7 \%$ de los triatominos fue positivo para este protozoario, y también se tipificó como Tcl. Además, se encontró que el mayor porcentaje de captura de triatominos correspondió a hembras $(56,2 \%)$ y se encontró una mayor frecuencia de sangre en 
el contenido intestinal de hembras $(33,7 \%)$ con respecto a los machos (5,6 \%).

Conclusiones. El presente estudio muestra que el genotipo circulante de $T$. cruzi tanto en ratas como en triatominos, provenientes del mismo área geográfica, corresponde a Tcl, lo cual permite sugerir que la rata doméstica ( $R$. rattus) es el huésped natural involucrado en el ciclo urbano de $T$. cruzi, y que $P$. geniculatus es el principal vector biológico implicado en la transmisión humana de la tripanosomiasis americana en Caracas.

\section{Emergencia de la enfermedad de Chagas en la amazonia boliviana: reporte de 14 casos agudos por transmisión oral de Trypanosoma cruzi en Guayaramerin-Beni, Bolivia}

José Santalla1, Tatiana Ríos¹, Patricia Oporto', Edy

Espinoza ${ }^{1}$, Aida Torrez ${ }^{2}$

1 Instituto Nacional de Laboratorios de Salud, Laboratorio de Parasitología, La Paz, Bolivia

2 Centro de Salud San Gabriel, Guayaramerin-Beni, Bolivia

Introducción. El Programa Nacional de Chagas de Bolivia reconoce seis departamentos endémicos, y no considera a ninguno de la amazonia; no se había reportado ningún caso de transmisión de Trypanosoma cruzi por vía oral en Bolivia.

En octubre de 2010 en la observación en el lugar de un grupo de muestras de gota gruesa y frotis de pacientes con sospecha de malaria de Guayaramerin-Beni, los microscopistas observaron formas flagelares; hubo confirmación en el Laboratorio de Parasitologia de INLASA y se determinó que se trataba de tripomastigotes sanguíneos de Trypanosoma cruzi por técnicas directas, aislamiento y biología molecular. Este hecho dio inicio a la investigación del primer brote de casos agudos de enfermedad de Chagas por transmisión oral en Bolivia.

Los objetivos del estudio fueron determinar el origen y la transmisión del brote de casos agudos de enfermedad de Chagas y comunicar las pruebas acumuladas del brote por transmisión oral.

Materiales y métodos. Se recibieron muestras de pacientes con síndrome febril inicial para determinación por técnicas directas e indirectas de la infección por $T$. cruzi.

Se hizo una investigación del brote para determinar las características epidemiológícas, entomológicas y parasitológicas. Se realizó tamización de la población bajo sospecha. Se buscaron todos los casos confirmados para recopilar información clínica y la variable común para determinar el origen del brote.

Resultados. De los 14 casos determinados, hubo 14 resultados positivos en el examen directo (microhematocrito); en la serología inicial, hubo 5 reacciones positivas y 9 no fueron reactivas; en la segunda serología, hubo 14 reactivas; por biología molecular (PCR) hubo 14 con ADN detectable.

En las observaciones clínicas: 14/14 presentaron un síndrome febril (temperatura mayor de 38 ${ }^{\circ} \mathrm{C}$; 10/13, linfocitosis; $11 / 13$ con transaminasas elevadas, y $11 / 13$ con bilirrubinas elevadas.

La tasa de ataque estimada para la OTB de San Miguel fue de $8 \%$.

Conclusiones. Se comprobó la infección por $T$. cruzipor una exposición a una fuente común, en este caso la fruta (majo) la cual se cosecha en el monte de diversos árboles, luego rudimentariamente se elabora un jugo de consumo local.

\section{Un modelo de inferencias de interacciones entre vectores y reservorios de Trypanosoma cruzi basado en la teoría de redes ecológicas}

C. N. Ibarra-Cerdeña ${ }^{1,2}$, L. Valiente-Banuet ${ }^{2}$, C. R. Stephens $^{2}$, J. M. Ramsey ${ }^{3}$

1 Instituto de Biología, Universidad Nacional Autónoma de México, México, D.F., México

2 Centro de Ciencias de la Complejidad, Universidad Nacional Autónoma de México, México, D.F., México

3 Instituto Nacional de Salud Pública, México, D.F., México

Introducción. Los triatominos son insectos hematófagos que al alimentarse de la sangre de mamíferos propician el ciclo de transmisión de Trypanosoma cruzi, causante de la enfermedad de Chagas. Aunque esta enfermedad ha recibido mucha atención desde el punto de vista clínico y epidemiológico, la investigación ecológica es muy escasa. El conocer los patrones de afinidad entre vectores y reservorios es crucial en el diseño de estrategias de control vectorial. Para que exista una interacción interespecífica, es necesario un patrón de ocurrencias simultáneas, así que se formuló la hipótesis que una fuerte codependencia en la distribución de vectores y mamíferos generaría una mayor probabilidad de interacción local.

Métodos. Esta hipótesis se evaluó con un enfoque de "minería de datos" utilizando bases de datos distribuidas e información empírica independiente. 
Con los valores significativos de épsilon, se generaron tablas de jerarquía para evaluar la predictibilidad de épsilon y se construyeron redes de interacción cuya topología fue analizada para detectar patrones de interacciones ecológicas.

Resultados. Se analizaron 2.967 localidades de recolección de 38 especies de triatominos y 62.629 de 499 especies de mamíferos con una métrica estandarizada recientemente (épsilon) como valor de vinculación geográfica. Los principales resultados fueron: 1) los mamíferos confirmados independientemente como reservorios de T. cruzi, tuvieron los valores más altos de épsilon; 2) los reservorios del primer cuartil fueron mamíferos sinantrópicos, y 3) se encontró una regionalización geográfica en los patrones de interacción. En este trabajo encontramos que la topología de la red de interacciones es una característica clave para la propagación de T. cruzi.

Conclusiones. Estos resultados indican que es necesario incorporar los mamíferos sinantrópicos en los planes de control vectorial peridoméstico.

\section{Chagas cardiomyopathy manifestations and Trypanosoma cruzi genotypes circulating in chronic chagasic patients from Colombia}

Juan David Ramírez¹, Lina Rendón¹, Fernando Rosas², José A. Marín-Neto ${ }^{3}$, Carlos Morillo ${ }^{4}$, Felipe Guhl ${ }^{1}$

1 Centro de Investigaciones en Microbiología y Parasitología Tropical, Universidad de los Andes, Bogotá, D.C., Colombia

2 Electrofisiología, Clínica Abood Shaio, Bogotá, D.C., Colombia

3 Cardiology Division, Universidad de São Paulo, São Paulo, Brasil

4 Cardiology Division, McMaster University, Ontario, Canada

Introduction. Chagas disease caused by Trypanosoma cruzi is a complex disease that is endemic and an important public health problem in Latin America. Trypanosoma cruzi has been classified in six discrete taxonomic units (DTU) based on the recently proposed nomenclature (Tcl, Tcll, TcIII, TcIV, TcV, and TcVI). The discovery of genetic variability within Tcl showed the presence of five genotypes (la, Ib, lc, Id and le) related to the transmission cycle of Chagas disease, Tcl is more prevalent in Colombia though Tcll has also been reported.

The objectives of this study were to determine the $T$. cruzi DTUs that are circulating in Colombian chronic chagasic patients, and to obtain more information about the molecular epidemiology of Chagas' disease in Colombia. We also assessed the presence of electrocardiographic, radiological and echocardiographical abnormalities with the purpose of correlating $T$. cruzi genetic variability and cardiac disease.

Methods and results. Molecular characterization was performed in Colombian adult chronic chagasic patients based on the intergenic region of the miniexon gene, the $24 \mathrm{Sa}$ and $18 \mathrm{~S}$ regions of rDNA and the variable region of satellite DNA, whereby the presence of $T$. cruzi I, II, III and IV was detected. In our population, mixed infections also occurred with Tcl-Tcll, Tcl-Tclll and Tcl-TcIV, as well as the existence of the Tcl genotypes showing the presence of genotypes la and Id. Patients infected with Tcl showed a higher prevalence of cardiac alterations than those infected with Tcll.

Conclusions. These results corroborate the predominance of $\mathrm{Tcl}$ in Colombia and are the first report of Tclll and TcIV in Colombian chagasic patients. Findings also indicate that Chagas' cardiomyopathy manifestations are more correlated with $\mathrm{Tcl}$ than with Tcll in Colombia. We concluded that $\mathrm{Tcl}$ is more related to cardiomyopathy than Tcll and we present the first report of Tclll and IV in chronic chagasic patients from Colombia. These results will help to elucidate the molecular epidemiology of $T$. cruzi in this country.

This work was published in PloS Neg. Trop. Dis. 2010

\section{Molecular ecoepidemiology and transmission dynamics of Trypanosoma cruzi I in Colombia}

Juan David Ramírez' ${ }^{1}$, Martin Llewellyn², Louisa Messenger ${ }^{2}$, Michael Miles², Felipe Guhl ${ }^{2}$

1 Centro de Investigaciones en Microbiologia y Parasitologia Tropical, Universidad de los Andes, Bogotá, D.C., Colombia

2 Pathogens Unit, London School of Hygiene and Tropical Medicine, London, United Kingdom

Introduction. Chagas disease caused by Trypanosoma cruzi is a complex disease that is endemic in Latin America. T. cruzi is classified into six discrete typing units (DTU). In Colombia, $\mathrm{Tcl}$ is more prevalent but Tcll has also been reported. The aim of this work was focused on elucidating the genetic structure of $T$. cruzi I using 269 singlecelled biological clones isolated from humans, insect vectors and reservoirs in Boyacá, Casanare and Santander provinces from Colombia in order 
to obtain information about the gene flow among domestic, peridomestic and sylvatic transmission cycles, searching for the plausible presence of recombination and/or introgression.

Materials and methods. Two hundred and sixty nine single-celled clones were genotyped as Tcl by direct sequencing of GPI gene. Twentyfour microsatellite markers were amplified in 269 single-celled clones and population genetics statistics were calculated. Also, 10 fragment maxicircle regions were sequenced in 100 clones according to the results of 24 STR. The sequences were aligned in MEGA 5.0 and concatenated according to the orientation of maxicircle molecule. A maximum composite likelihood reconstruction was developed with 1,000 iterations and a NJ DAS tree with the microsatellite data. A discriminant analysis of principal components (DAPC) analysis was developed in order to observe population subdivision and plausible events of introgression between nuclear and mithocondrial markers.

Results and conclusions. We were able to observe recombination based on maxicircle DNA data and introgression between nuclear and mithocondrial DNA using the DAPC strategy. We found recombination at a low scale but this suggests that $T$. cruzi reproduction is not merely clonal and sexual recombination is a valuable key in the diversification of $T$. cruzi. We suggest that $T$. cruzi structure is a composite of likely recombination events as has been evidenced in other parasitic protozoa. We were able to identify one clearly genotype associated to human infection corroborating previous hypothesis using other molecular markers. Our results corroborate the high genetic diversity within $\mathrm{Tcl}$ and the need to develop further research in order to establish the real implications of this DTU in the disease outcome.

$$
-\bullet
$$

\section{Inmunofisiopatogenia de la infección por vía oral y vía congénita por Trypanosoma cruzi}

Laura Viviana Sánchez ${ }^{1}$, Juan David Ramírez ${ }^{2}$

1 Facultad de Medicina, Universidad Militar Nueva Granada, Bogotá, D.C., Colombia

2 Centro de Investigaciones en Microbiología y

Parasitología Tropical, Universidad de los Andes,

Bogotá, D.C., Colombia

Introducción. La infección por Trypanosoma cruzi puede ocurrir por vía oral y por vía vertical, dos mecanismos poco estudiados.

El objetivo del presente trabajo fue revisar los elementos determinantes de la respuesta inmunitaria del tubo digestivo y de la barrera materno- placentaria, sus mecanismos de reconocimiento y respuesta, y los elementos de $T$. cruzi necesarios para el establecimiento de la infección, para deducir las diferentes vías de señalización y las moléculas involucradas en estas dos vías de trasmisión.

Metodología. Se buscó información en la base de datos PubMed, con los términos "Inmunología/ fisiología del tracto gastrointestinal", "inmunología/ fisiología del embarazo", "Infección de Trypanosoma cruzi por vía oral", "enfermedad de Chagas congénita", "respuesta inmune a la infección por $T$. cruzi" y de otros términos cuando se necesitó ampliar el conocimiento sin restricciones de fecha o idioma; se incluyó información de publicaciones de la OMS, la OPS, el TDR y la FIOCRUZ.

Resultados. El reconocimiento de $T$. cruzi en la infección congénita se da por los TLR 2, que reconocen mucinas acopladas al glucofosfatidilinositol y la proteína Tc52, y los TLR 4 que reconocen los fosfolípidos glucoinositol, expresados en los citotrofoblastos vellosos y los trofoblastos extravellosos que inducen un perfil TH1, producción de inmunoglobulinas capaces de activar el complemento y aumentanla producción de Fgl2 (protrombinasa inductora de la formación de coágulos) e inducen la producción de interferón de tipo 1 y la activación de GTPasas relacionadas con la inmunidad, respectivamente. En la infección por vía oral participan los TLR 2, 4, 5 y 9 de las células epiteliales estomacales y la activación dependiente de calmodulina/calcineurina del factor de transcripción NFATc1 mediada por las glucoproteínas 30,82 , cruzipaína y la oligopeptidasa B.

Conclusiones. Trypanosoma cruzi presenta gran diversidad bioquímica y genética, por lo cual la respuesta inmune del huésped presenta gran variabilidad según la cepa infectante, el estado inmunológico del paciente y la vía de infección.

\section{Caracterización clínica y serológica de la enfermedad de Chagas en una comunidad indígena de la Sierra Nevada de Santa Marta}

María Teresa Mojica ${ }^{1}$, Jorge Armando Egurrola ${ }^{1}$,

Duámaco Escribano ${ }^{2}$, Juan Carlos Dib²

1 CIMET, Centro de Investigación en Medicina Tropical, Universidad del Magdalena, Santa Marta, Colombia

2 CIET, Centro de Investigación en Enfermedades Tropicales, Fundación Salud para el Trópico, Santa Marta, Colombia

Introducción. El presente estudio se realizó en la comunidad indígena Umandita, vertiente norte de 
la Sierra Nevada de Santa Marta, considerada una de las regiones más endémicas para la enfermedad de Chagas en Colombia.

Materiales y métodos. A 185 personas de la comunidad se les aplicó una encuesta epidemiológica, historiaclínicayencuestaserológica utilizando la prueba rápida para Chagas (Chagas Stat Pack TM de Chembio) como tamización, ELISA-IgG (Chagatek Elisa Biomerieux) e IFI-IgG como pruebas confirmatorias.

Resultados. El $56 \%$ de los pacientes seropositivos (14/25) cursaban asintomáticos. En 44 \% (11/25) de la población sintomática, el dolor torácico fue el principal síntoma referido, seguido de síncope, $23 \%$ (5/25); se observó que $84 \%(21 / 25)$ cursaba con examen físico normal y $16 \%(4 / 25)$ tenía alguna alteración en el examen físico; de los pacientes seropositivos sintomáticos (11/25), sólo tres presentaron alguna alteración en el examen físico. En la población seronegativa no hubo variación significativa con relación a la presencia de sintomatología. Para el examen físico anormal la relación entre individuos infectados y no infectados con $T$. cruzi fue de 4:1. Todos los pacientes seropositivos presentaron, al menos, uno de los antecedentes epidemiológicos de riesgo.

Conclusiones. Las características socioeconómicas y culturales de la población estudiada y las características ecológicas del área hacen muy difícil un programa de control vectorial sostenido. Es probable que esta población que, al parecer, presenta infección reciente y la mayoría se encuentra en fase latente o crónica incipiente, pueda beneficiarse del tratamiento y en caso de no alcanzar la curación, mejorar el pronóstico evitando el progreso de la cardiopatía.

También se sugiere poner más atención a la comunidad y sus costumbres, con el fin de diseñar estrategias de prevención y control más eficaces.

Financiado por COLCIENCIAS contrato 308-2007 - $\bullet$

\section{Seroepidemiología de la infección por Trypanosoma cruzi en perros, en el estado Sucre, Venezuela}

Mariolga Berrizbeitia ${ }^{1,2}$, Juan Luis Concepción ${ }^{3}$, Valentina Carzola ${ }^{4}$, Jéssicca Rodríguez ${ }^{1}$, Segundo Quiroz ${ }^{5}$, Belzaira Rivas 5

1 Posgrado en Biología Aplicada, Núcleo de Sucre, Universidad de Oriente, Venezuela.

2 Instituto de Biomedicina y Ciencias Aplicadas, Universidad de Oriente, Venezuela.

${ }^{3}$ Laboratorio de Enzimología de Parásitos, Universidad de los Andes, Venezuela
4 Departamento de Bioanálisis, Universidad de Oriente, Venezuela

5 Centro de Asesorías y Proyectos Estadísticos, Escuela de Estadística, Universidad de Los Andes, Venezuela

Introducción. Los perros facilitan y mantienen la transmisión de la infección por Trypanosoma cruzi ya que constituyen una fuente de alimentación segura para vectores triatominos.

El objetivo de este estudio fue determinar la seroepidemiología de la infección por T. cruzi en perros en 15 municipios del estado Sucre, Venezuela.

Materiales y métodos. Se utilizó un diseño muestral por conglomerados, bietápico. Las unidades primarias de muestreo fueron los centros poblados en el área rural y las unidades secundarias, las viviendas. Las muestras de suero de los perros se evaluaron utilizando el kit CruziELISA (Laboratorio de Enzimología de Parásitos, ULA, Venezuela) y por la prueba de ligación de múltiples antígenos (MABA). Igualmente, se aplicaron encuestas epidemiológicas para evaluar factores de riesgo asociados a la infección.

Resultados. Se estudiaron los 15 municipios que componen el estado Sucre, 96 centros poblados rurales y los perros que habitaban en 576 viviendas. Se evaluaron 363 perros, el promedio de la edad fue de $31,65 \pm 25,87$ meses (rango, 2 a 144 meses), 226 eran machos y 137 hembras. La combinación de las pruebas ELISA/MABA detectó 78 sueros positivos, 275 negativos y 10 resultados inconclusos. Por lo tanto, la seroprevalencia de la infección para $T$. cruzi en perros en el estado Sucre fue de 21,49\%. No se encontró asociación estadística significativa entre la infección por $T$. cruzi en perros y la costumbre de los animales de cazar, dormir al aire libre y deambular libremente por el centro poblado, o por el sexo del animal. Igualmente no se encontró asociación con ciertos hábitos alimentarios, como comer ratas o animales silvestres.

Conclusiones. La alta seroprevalencia detectada en los perros muestra que en esta región de Venezuela existe un factor de riesgo importante de transmisión de este parásito a poblaciones humanas.

\section{Prevalencia de anticuerpos contra Trypanosoma cruzi en perros domésticos del área rural del municipio de Garagoa, Boyacá}

Nataly Marcela Garzón, Diego Alexander ManriqueAbril, Fred G. Manrique-Abril 
Centro de Investigación Epidemiológica de Colombia y Grupo de investigación en Salud Pública, Universidad

Pedagógica y Tecnológica de Colombia, Tunja,

Colombia

Introducción. Los perros domésticos son los principales reservorios de infecciones humanas por parásitos como Trypanosoma cruzi, y desempeñan un papel importante en la epidemiología de la enfermedad de Chagas. Los problemas de salud pública asociados con $T$. cruzi revelan la necesidad de investigación de estas infecciones en perros que puedan ser fuentes de posterior transmisión del parásito a los seres humanos. Es esencial obtener información de la prevalencia y la distribución geográfica de las infecciones en perros para el desarrollo y seguimiento de las medidas de control estratégico, para determinar factores que aumentan el riesgo de infección en animales o en humanos, y para hallar los principales signos clínicos que el médico veterinario puede percibir en la presentación de un caso y así, ser parte de la vigilancia epidemiológica de la enfermedad de Chagas.

El objetivo del estudio fue determinar la prevalencia de anticuerpos contra $T$. cruzi en perros domésticos del área rural de Garagoa.

Materiales y métodos. Se definieron las veredas donde se ha reportado con mayor incidencia la enfermedad de Chagas en humanos. Luego se procedió a averiguar las personas que poseían perros en los hogares y se procedió con la visita y la toma de las muestras.

Las muestras de sangre de perros se obtuvieron por punción de capilares sanguíneos de la oreja o directamente de la vena cefálica del brazo. Se hizo la prueba con tiras (Inbios, Seattle, WA), según las instrucciones de manufactura. También se aplicó a las personas residentes en estos hogares, una encuesta que exploraba aspectos demográficos, sanitarios y etológicos de los animales, que sirvieron para evaluar posibles relaciones con la infección.

Resultados. Se encontró una prevalencia de 8,8 \% de 57 muestras obtenidas La distribución de resultados seropositivos fue mayor en animales criollos; se observó mayores resultados seropositivos en viviendas de madera para el animal.

Hubo mayor reacción en machos que en hembras pero sin diferencias estadísticas $(p>0,05)$; todos los casos positivos se presentaron en animales mayores de 9 años. Las variables fisiológicas de los animales no presentaron cambios significativos entre los positivos y los negativos; esto tiene relevancia dado que la enfermedad se presenta silenciosa sin signos patognomónicos de la enfermedad.

En todos los sitios reconocieron al pito como transmisor de la enfermedad de Chagas. La tenencia de otros animales se reportó en el 20,8 $\%$ de los perros positivos, convivían con aves; el resto se reportó en animales negativos.

La desparasitación y vacunación como medida sanitaria en los animales es un factor protector para los resultados seropositivos de enfermedad de Chagas con significancia estadística $(p \leq 0,05)$. Conclusiones. La prevalencia reportada, aunque baja, es un hallazgo que constituye una alerta epidemiológica para emprender medidas de control y prevención mediante tamizaciones intensivas y educación a las comunidades sobre los riesgos de contagio que se incrementan para las personas que conviven con mascotas caninas en franca cercanía debido a que los vectores tienen una fuente de alimentación auxiliar importante en periodos en que no hay población humana presente.

El perro aumenta el riesgo de infección de los vectores, y de ésta, la transmisión al hombre. Los perros resultan excelentes centinelas para la vigilancia de programas de control vectorial.

\section{Miocardiopatía chagásica infantil en México, resultados preliminares}

Paz María Salazar-Schettino ${ }^{1}$, Martha I. Bucio-Torres ${ }^{1}$, Margarita Cabrera-Bravo ${ }^{1}$, Adela Ruiz-Hernández¹, Carlos Zamora-González², Clara Vázquez-Antona², David Bialostozky²

1 Laboratorio de Biología de Parásitos, Departamento de Microbiología y Parasitología, Facultad de Medicina, Universidad Nacional Autónoma de México, México, D.F., México

2 Instituto Nacional de Cardiología "Dr. Ignacio

Chávez", SSA, México, D.F., México

Introducción. En México se desconoce la prevalencia real de la enfermedad de Chagas; la infección por Trypanosoma cruzi en población infantil permite determinar la transmisión activa. Para evaluar la magnitud de la enfermedad en esta población y realizar medidas de intervención y vigilancia epidemiológica, se requiere caracterizar el perfil epidemiológico, clínico y cardiológico, es decir, identificación de riesgos, signos, síntomas y anormalidades en el electrocardiograma, el ecocardiograma y la ventriculografía radioisotópica en equilibrio.

Materiales y métodos. Se estudiaron individuos menores de 18 años seropositivos (ELISA e IFI), 
provenientes de tres estados. Se aplicó cuestionario e historia clínica, se obtuvo electrocardiograma, ecocardiograma y ventriculografía radioisotópica en equilibrio.

Resultados. Se identificaron 49 menores seropositivos: 8 (16\%) de Querétaro, 37 (76 \%) de San Luis Potosí y 4 (8\%) de Veracruz, de los cuales, 46 (94\%) identificaron al triatomino; 13 (26 \%) vivían en casa; 16 (33\%), en jacal, y 20 (41\%) en choza. Al interrogatorio, 13 (27 \%) estaban asintomáticos y 36 (73 \%) refirieron disnea, palpitaciones, taquicardia o dolor precordial. El electrocardiograma determinó 21 (43\%) con trazo normal y 28 (57\%) con trazos compatibles con bloqueo incompleto o incipiente de rama derecha del haz de His y bradicardia sinusal. La ecocardiografía mostró como alteraciones principales, engrosamiento del tabique, disminución de la fracción de expulsión del ventrículo izquierdo, hipertensión pulmonar y alteraciones en la movilidad segmentaria. En la ventriculografía radioisotópica en equilibrio se encontró un patrón más temprano de contracción.

Conclusiones. Está en proceso la correlación clínico-epidemiológica con el análisis de electrocardiografía, ecocardiografía y ventriculografía radioisotópica en equilibrio. Un alto porcentaje identificó al transmisor y habitaban viviendas consideradas de riesgo para la infección. En caso de existir trazos electrocardiográficos normales, la ecocardiografía es un recurso diagnóstico importante, especialmente en casos asintomáticos y trazos electrocardiográficos normales, para la detección de lesiones incipientes, especialmente en edad pediátrica. Las alteraciones ecocardiográficas fueron más frecuentes en pacientes provenientes de Querétaro. En la ventriculografía radioisotópica en equilibrio existía una activación temprana de ambos ventrículos.

\section{Enfermedad de Chagas en mujeres latinoamericanas embarazadas y transmisión congénita en una zona no endémica: la experiencia de un programa de salud de la Comunidad Valenciana (España)}

R. Borras ${ }^{1}$, C. Barona-Vilar², M. J. Giménez-Martín ${ }^{3}$, T. Fraile $^{4}$, C. González-Steinbauer ${ }^{2}$, C. Parada ${ }^{5}$, A. GilBrusola $^{3}$, D. Bravo', D. Navarro ${ }^{1}$, M. D. Gómez ${ }^{3}$, A. PérezTamarit $^{6}$, L. Fernández-Silveira ${ }^{6}, A$. Fullana-Montoro²

1 Departamento de Microbiología, Facultad de Medicina y Hospital Clínico Universitario,

Valencia, España
2 Centro Superior de Investigación en Salud Pública, Valencia, España

3 Servicio de Microbiología, Hospital Universitario y Politécnico La Fe, Valencia, España

4 Servicio de Microbiología, Consorcio Hospital General Universitario, Valencia, España

5 Centro de Transfusión, Valencia, España

6 Servicio de Pediatría, Hospital Universitario y

Politécnico La Fe, Valencia, España

Introducción. La enfermedad de Chagas se ha convertido en un problema emergente de salud pública en España debido al elevado número de inmigrantes procedentes de países endémicos, de los que el 2,5\% podría estar infectado por Trypanosoma cruzi. En 2009, en la Comunidad Valenciana se diseñó un protocolo para el diagnóstico precoz de la enfermedad en mujeres embarazadas de países endémicos y sus hijos recién nacidos.

Los objetivos de este estudio han sido: i) evaluar la implementación del protocolo; ii) determinar la prevalencia de la enfermedad, y iii) conocer la tasa de transmisión vertical.

Material y métodos. Se trata de un estudio transversal (2009-2010), realizado con los datos obtenidos de los sistemas de información de los Servicios de Microbiología de tres hospitales universitarios. La población fueron las mujeres embarazadas de países endémicos, reclutadas durante el primer trimestre del embarazo por las comadronas, y los neonatos de madres enfermas. Loscriteriosdiagnósticofueron:i)mujeres:presencia de anticuerpos anti-T. cruzi (recomendaciones de la OMS); ii) neonatos: microhematocrito, IgM anti-T. cruzi o PCR (por duplicado) positivos, o persistencia de $\lg G\left(\geq 7^{\circ}\right.$ mes).

Resultados. La proporción de mujeres estudiadas fue de $95,4 \%$ (1.975/2.070) y la prevalencia global fue de $11,4 \%$. La enfermedad fue más común entre las suramericanas que entre las mejicanas y centroamericanas (11,8\% Vs. $2,5 \%$; $p<0,01)$, y en las procedentes del Cono Sur que en las oriundas de la Región Andina (24,8 \% Vs. 0,1%; p<0,0001); todos los casos del Cono Sur procedían del Gran Chaco. El 94,7 \% de los casos eran mujeres bolivianas. La transmisión vertical se detectó en ocho (3,7\%) neonatos de madres bolivianas.

Conclusiones. i) La implementación del programa ha sido exitosa; ii) el $11,4 \%$ de la población está afecta de enfermedad de Chagas y ésta es significativamente más común entre la población suramericana, especialmente, la boliviana, y iii) la transmisión congénita se ha dado exclusivamente entre los hijos de madres bolivianas. 


\section{Enfermedad de Chagas y sus factores de riesgo en mujeres gestantes de la zona endémica de Santander, Colombia, análisis preliminar}

Yeny Z. Castellanos ${ }^{1}$, Zulma M. Cucunubá2, Luis C. Orozco ${ }^{1}$, Astrid C. Flórez², Lyda Muñoz ${ }^{2}$, Paula Pavía $^{3}$, Marleny Montilla ${ }^{2}$, Rubén Santiago Nicholls ${ }^{2}$, Concepción J. Puerta ${ }^{3}$

1 Universidad Industrial de Santander, Bucaramanga, Colombia

2 Grupo de Parasitología, Instituto Nacional de Salud, Bogotá, D.C., Colombia

3 Laboratorio de Parasitología Molecular, Pontificia Universidad Javeriana, Bogotá, D.C., Colombia

Introducción. EnColombiaseadelantan programas para el control vectorial y por transfusión de la enfermedad de Chagas, no obstante, se desconoce la magnitud de la transmisión congénita.

El objetivo del estudio fue determinar la prevalencia de infección por Trypanosoma cruzi en mujeres gestantes de la zona endémica para la enfermedad de Chagas en Santander.

Materiales y métodos. De julio de 2010 a julio de 2011 se incluyeron en el estudio mujeres gestantes de 22 municipios de tres provincias de Santander, a través de los programas de control prenatal. Previo consentimiento informado, se tomó muestra y se realizó detección de anticuerpos IgG anti- $T$. cruzi, mediante pruebas ELISA, IFI y HAI. Se consideraron como casos confirmados cuando habían sido positivos por, al menos, dos pruebas reactivas. Mediante cuestionario previamente validado, se realizó el análisis de los factores de riesgo. Se determinaron las prevalencias de infección global y por provincias. Las estimaciones de factores de riesgo se realizaron mediante razones de prevalencia. Los intervalos de confianza del 95 $\%\left(\mathrm{IC}_{95 \%}\right)$ fueron considerados estadísticamente significativos.

Resultados. Se encontró una prevalencia global de $3,2 \%\left(I_{95 \%} 2,4-4,3\right)$ entre 1.390 mujeres gestantes que fueron incluidas. La prevalencia por provincias fue: Guanentina, 5,7 \% ( $\left.\mathrm{IC}_{95 \%} 3,9-8,3\right)$; García Rovira: 3,2 \% (IC ${ }_{95 \%} 1,7$ - 5,7), y Comunera: $1,0 \%\left(\mathrm{IC}_{95 \%} 0,4-2,4\right)$. Los principales factores de riesgo relacionados fueron: edad mayor de 29 años, $\mathrm{RP}=6,2$ (IC IC ${ }_{95 \%} 1,9$ - 20,5); conocimiento previo del vector: 5,6 (IC IC I5\% $_{2,6}$ - 11,9), y haber estado en contacto con el vector alguna vez en la vida, $R P=10,0$ (IC IC ${ }_{95 \%} 5,5$ - 18,1).

Conclusiones. Por primera vez se determinó la prevalencia de la enfermedad de Chagas en mujeres gestantes de Santander. Los factores de riesgo más importantes están relacionados con la probabilidad de contacto con el vector. Es necesario hacer seguimiento de los neonatos de estas mujeres gestantes durante el primer año de vida para descartar o confirmar un caso de Chagas congénito. 